\title{
Lipase-catalyzed resolution of anti-6-substituted 1,3-dioxepan-5-ols
}

\author{
Michelangelo Gruttadauria, ${ }^{*}$ Paolo Lo Meo, Serena Riela, \\ Francesco Giacalone and Renato Noto \\ Dipartimento di Chimica Organica 'E. Paternò', Università di Palermo, Viale delle Scienze, \\ Parco d'Orleans, Pad. 17, I-90128 Palermo, Italy \\ Received 5 October 2006; accepted 15 November 2006 \\ Available online 12 December 2006
}

\begin{abstract}
Several anti-6-substituted 1,3-dioxepan-5-ols were kinetically resolved using an immobilized lipase (Amano PS-C II) in toluene in the presence of vinyl acetate at $30^{\circ} \mathrm{C}$. This approach provided, in some cases, the alcohol and the acetate in high enantiomeric purity, depending on the nature of the substituent $\left(\mathrm{R}=\mathrm{N}_{3}, \mathrm{SePh}, \mathrm{I}, \mathrm{OBn}\right)$ and the acetal group (unsubstituted or dimethyl). The role of the size of substituents is also discussed. Enantiopure anti-6-substituted 1,3-dioxepan-5-ols are useful building blocks.

(C) 2006 Elsevier Ltd. All rights reserved.
\end{abstract}

\section{Introduction}

The importance of chiral 1,2-amino alcohols is evident since they are often present in many biologically active compounds. Enantiomerically pure syn- and anti-2-amino1,3,4-trihydroxy-butane $\mathbf{1}$ and syn- and anti-2-azido1,3,4-trihydroxy-butane 2 (Fig. 1) would be interesting chiral building blocks in the synthesis of several important compounds such as sphingosines, ${ }^{1}$ FR900482 an antitumour antibiotic, ${ }^{2}$ kainic acid, ${ }^{3}$ liposidomycins ${ }^{4}$ and glycomimetics. ${ }^{5}$ Particularly, such compounds can be used for the synthesis of $\mathrm{C} 4$ unit of Nelfinavir, one of the most potent HIV-protease inhibitors (Fig. 2). ${ }^{6}$
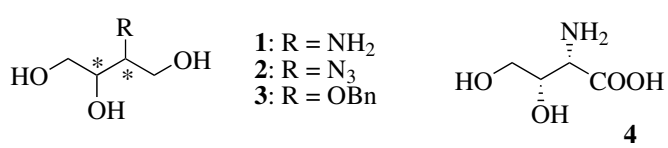

Figure 1.

Furthermore, $(2 S, 3 S)$-2-amino-3,4-dihydroxybutyric acid 4 has been used as a synthetic intermediate in the synthesis of $\beta$-lactam antibiotics and phytosiderophores. ${ }^{7}$

These compounds can be obtained from the chiral pool. Enantiomerically enriched derivatives of $\mathbf{1}$ have already

\footnotetext{
* Corresponding author. Tel.: +39 091 596919; fax: +39 091 596825; e-mail: mgrutt@unipa.it
}

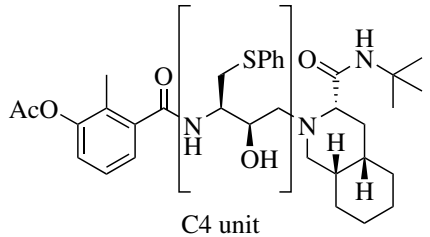

Figure 2. Structure of Nelfinavir.

been obtained from transformations of isoascorbic acid, ${ }^{1 \mathrm{~d}, 4 \mathrm{c}}$ ascorbic acid, ${ }^{4 \mathrm{~b}, 5 \mathrm{~b}}$ erythrulose ${ }^{8}$ and from $\mathrm{D}$ - or L-diethyl tartrate. ${ }^{1 \mathrm{a}}$

Compounds 1 can also be prepared from chiral aziridino alcohols obtained by enzymatic method, ${ }^{9}$ from separation of a diastereomeric mixture of trans-amino alcohol obtained by meso-epoxide ring opening with chiral amine, ${ }^{6}$ from asymmetric aminolysis of meso-epoxide using Ti(S)-1,1'-bi-2-naphthoxide complex as chiral catalyst, ${ }^{10}$ from monoprotected optically active cis-1,4-dihydroxy2,3-butene oxide. ${ }^{11}$ The latter could be generated through Sharpless asymmetric epoxidation. ${ }^{11}$ Sharpless asymmetric aminohydroxylation has also been used employing 2-butene-1,4-diol derivatives as substrates. ${ }^{12}$

In addition to compounds $\mathbf{1}$ and $\mathbf{2}$, compound $\mathbf{3}$ is a very interesting building block too. It can be prepared starting from the chiral pool using L- or D-tartrate for $(S, S)-{ }^{13}$ or $(R, R)$-enantiomers ${ }^{14}$ or from $\mathrm{D}$-erythronolactone ${ }^{15}$ both for $(R, S)$ - and $(S, R)$-enantiomers. 
In connection with our interest in stereoselective synthesis using lipase resolution of secondary alcohols, organocatalysis and organoselenium compounds, ${ }^{16}$ we were interested in the stereoselective preparation of compounds 1-3 through enzymatic resolution. Enzymatic resolution of syn-2-azido-1,3,4-trihydroxy-butane catalyzed by lipases, both in the transesterification mode ${ }^{17}$ and in the hydrolysis mode, ${ }^{18}$ have been reported.

Enzymatic resolution in the transesterification mode gave compound $\mathbf{5}$ in 33\% yield ( $>99 \%$ ee) and compound $\mathbf{6}$ in $19 \%$ yield $(94 \%$ ee), whereas enzymatic resolution in the hydrolysis mode gave compound 7 in 30\% yield ( $>99 \%$ ee). These approaches gave high ee values, but low isolated yields (Fig. 3).<smiles>CC(=O)OCC(N)C(O)COC(C)=O</smiles><smiles>CC(=O)OC[C@@H](N)[C@H](COC(C)=O)OC(C)=O</smiles><smiles>CC(=O)OC[C@@H](CO)[C@@H](C#N)OC(C)=O</smiles>

Figure 3.

Herein, we report our results on investigations about kinetic resolution, mediated by an immobilized lipase (Amano PS-C II), of several racemic anti-6-substituted 1,3dioxepan-5-ols as useful precursors of compounds 1-3.

\section{Results and discussion}

Compounds $( \pm)$-anti-8a-i (Table 1) were easily prepared starting from the proper cyclic epoxide by ring opening with $\mathrm{NaN}_{3}, \mathrm{NaSePh}, \mathrm{NaI}$ or $\mathrm{BnOH}$.

Table 1. The target molecules

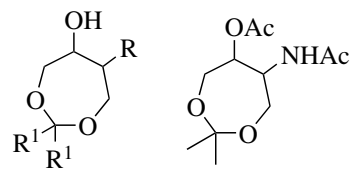

$( \pm)$-anti-8a-i

$( \pm)$-anti-9

\begin{tabular}{lll}
\hline $\mathbf{8}$ & $\mathrm{R}$ & $\mathrm{R}^{1}$ \\
\hline $\mathbf{a}$ & $\mathrm{N}_{3}$ & $\mathrm{Me}$ \\
$\mathbf{b}$ & $\mathrm{NHCbz}$ & $\mathrm{Me}$ \\
$\mathbf{c}$ & $\mathrm{NHCOPh}$ & $\mathrm{Me}$ \\
$\mathbf{d}$ & $\mathrm{N}_{3}$ & $\mathrm{H}$ \\
$\mathbf{e}$ & $\mathrm{SePh}$ & $\mathrm{Me}$ \\
$\mathbf{f}$ & $\mathrm{SePh}$ & $\mathrm{H}$ \\
$\mathbf{g}$ & $\mathrm{I}$ & $\mathrm{H}$ \\
$\mathbf{h}$ & $\mathrm{OCH}_{2} \mathrm{Ph}$ & $\mathrm{Me}$ \\
$\mathbf{i}$ & $\mathrm{OCH}_{2} \mathrm{Ph}$ & $\mathrm{H}$ \\
\hline
\end{tabular}

We started our investigation with compound $( \pm)$-8a using PS-C II lipase in toluene in the presence of vinyl acetate. The resolution of $( \pm)$-8a was slow. After $24 \mathrm{~h}$, the conversion was $26 \%$ and the ee value of ester 11a was $92 \%$ (Table 2 , entry 1 ). After $48 \mathrm{~h}$ the conversion was $35 \%$ and the ee value of 11a was $90 \%$ (entry 2). The reaction was stopped after $240 \mathrm{~h}$ and the conversion was $52 \%$. Alcohol 10a was obtained in $94 \%$ ee and ester 11a in $87 \%$ ee (entry 3 ). We carried out the reaction again and stopped it when alcohol 10a was enantiomerically pure $(57 \%$ conversion, entry 4$)$. Resolution in THF gave a lower conversion than in toluene after $24 \mathrm{~h}$ (entry 5). Resolution in THF gave similar results, about ee values, at higher conversion (entry 6). Using Candida antarctica lipase (Novozym 435), no selectivity was observed after $24 \mathrm{~h}$ (4\% ee).

Table 2. Enantioselective lipase-catalyzed kinetic resolutions of compounds $( \pm)-\mathbf{8} \mathbf{a}-\mathbf{i}^{\mathrm{a}}$

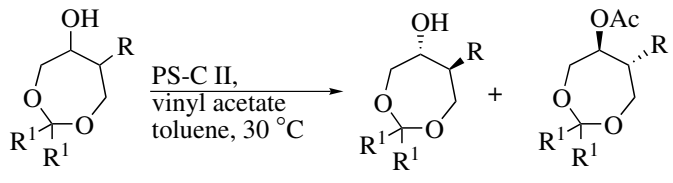

$( \pm)$-anti-8a-i 10a-i $\quad 11$ a-i

\begin{tabular}{ccccccc}
\hline Entry & Compd & $t(\mathrm{~h})$ & $c(\%)$ & ee $\mathbf{1 0}(\%)$ & ee $\mathbf{1 1}(\%)$ & $E^{\mathrm{b}}$ \\
\hline 1 & $( \pm)-\mathbf{8 a}$ & 24 & 26 & 32 & 92 & 38 \\
2 & & 48 & 35 & 48 & 90 & 31 \\
3 & & 240 & 52 & 94 & 87 & 51 \\
4 & & 288 & 57 & $>99$ & 76 & 37 \\
$5^{\mathrm{c}}$ & & 24 & 14 & 16 & 99 & $>200$ \\
$6^{\mathrm{c}}$ & & 168 & 42 & 65 & 90 & 37 \\
7 & $( \pm)-\mathbf{8 b}$ & $-\mathrm{d}$ & - & - & - & - \\
8 & $( \pm)-\mathbf{8 c}$ & $-\mathrm{e}$ & - & - & - & - \\
9 & $( \pm)-\mathbf{8 d}$ & 3.5 & 51 & $>99$ & 96 & $>200$ \\
10 & $( \pm)-\mathbf{8} \mathbf{e}$ & 168 & 37 & 58 & $>99$ & $>200$ \\
11 & & 264 & 50 & $>99$ & $>99$ & $>200$ \\
12 & $( \pm)-\mathbf{8 f}$ & 1 & 50 & $>99$ & $>99$ & $>200$ \\
13 & $( \pm)-\mathbf{8 g}$ & 2 & 44 & 70 & 90 & 40 \\
14 & $( \pm)-\mathbf{8 h}$ & 0.5 & 50 & 14 & 14 & 1.5 \\
15 & $( \pm)-\mathbf{8 i}$ & 2 & 50 & $>99$ & $>99$ & $>200$ \\
\hline
\end{tabular}

${ }^{a}$ Conditions: $( \pm)-8(0.4 \mathrm{mmol})$, lipase $(50 \mathrm{mg})$, vinyl acetate (3 equiv), toluene $(0.9 \mathrm{~mL}), 30^{\circ} \mathrm{C}$.

${ }^{\mathrm{b}} E=\ln [1-c(1+\mathrm{ee}(\mathbf{1 1}))] / \ln [1-c(1-\operatorname{ee}(\mathbf{1 1}))], c=\operatorname{ee}(\mathbf{1 0}) /[\operatorname{ee}(\mathbf{1 0})+\operatorname{ee}(\mathbf{1 1})]$.

${ }^{\mathrm{c}}$ Solvent: THF.

${ }^{\mathrm{d}}$ Decomposition.

${ }^{\mathrm{e}}$ No reaction.

In order to improve the enantiopreference, we decided to follow two different strategies. The first was to study the enzymatic resolution of compounds $( \pm)$-8b,c having a larger $\mathrm{R}$ group than azido group and $\mathrm{R}^{1}=\mathrm{Me}$. The second one was to keep the azido substituent $\left(R=N_{3}\right)$ and to put a smaller acetal substituent $\left[\mathrm{R}^{1}=\mathrm{H} ;( \pm)\right.$-8d]. We also prepared $N, O$-diacetyl compound $\mathbf{9}$ in order to study its alcoholysis. ${ }^{19}$

First we prepared the Cbz derivative $( \pm)-\mathbf{8 b}$, but no enzymatic resolution was carried out since this product gave, after $1 \mathrm{~h}$, a complex and inseparable mixture. ${ }^{6 \mathrm{~b}}$ No resolution was observed with compound $( \pm)-\mathbf{8 c}$. Also the conversion of $\mathrm{N}, \mathrm{O}$-diacetyl compound $( \pm)-9$ was very poor even after several days.

We then turned our attention to compound $( \pm)-8 d$. The resolution of this molecule was very fast compared to $( \pm)$-8a. Indeed, after $3.5 \mathrm{~h}$ we obtained a $51 \%$ conversion. Moreover, the enantiopreference was high $(E>200)$ giving ester 11d in $96 \%$ ee and alcohol 10d in $>99 \%$ ee (entry 9 ). 
cis-1,2-Amino alcohols can be prepared starting from anti$\beta$-hydroxy selenides through displacement of the phenylselenonyl group, following the procedure developed by Tiecco. ${ }^{20}$ For this reason, we investigated the enzymatic resolution of compound $( \pm)-8 \mathbf{e}$.

Compound $( \pm)-8 \mathrm{e}$ reacted slowly. The reaction was complete after $264 \mathrm{~h}$. However, the resolution was excellent ( $>99 \%$, entry 11). This result confirmed our hypothesis that with larger $\mathrm{R}$ groups better ee values could be reached. However, the reaction times are long.

Resolution of the less hindered compound $( \pm)$-8f gave enantiopure alcohol $\mathbf{1 0 f}$ and ester $\mathbf{1 1 f}$ in a very short time (1 h, entry 12). In order to have a different leaving group, we decided to use an iodine atom instead of the phenylseleno group. Since an iodine atom is similar in size to the azido group, we investigated resolution of compound $( \pm)-8 g$, which as compound $( \pm)-8 d$, does not have the bulkier dimethyl acetal group. In this case a lower $E$ value compared with compound $( \pm)-\mathbf{8 d}$ was obtained. Indeed, after a $44 \%$ conversion ester 11g was obtained in $90 \%$ ee (entry 13). The use of other lipases, such as C. antarctica lipase (Novozym 435) or Candida rugosa lipase, gave poor results. For instance, with the latter lipase, the ee value of $\mathbf{1 1 g}$ was $30 \%$ after a $24 \%$ conversion.

Resolution of benzyloxy derivative $( \pm)$-8h gave an unexpected result (entry 14). Indeed, the reaction was very fast, being complete in $30 \mathrm{~min}$ and giving both alcohol $\mathbf{1 0 h}$ and ester $11 \mathrm{~h}$ with very low ee value $(14 \%)$. In order to improve the enantiopreference, we carried out the resolution of compound $( \pm)-\mathbf{8 i}$. In this case, as for $( \pm)-\mathbf{8 d}$ and $( \pm)-\mathbf{8 f}$, the enantiopreference was excellent giving both enantiomeric pure alcohol 10i and ester 11i (entry 15).

The enantiomeric excess values were determined by HPLC using a Chiracel OD-H chiral column and $n$-hexane/ $i$-propanol as eluent (Table 3).

Table 3. HPLC retention times of compounds 10a-i and 11a-i

\begin{tabular}{lcllll}
\hline Compd & $t_{\mathrm{R}}(\mathrm{min})$ & Eluent $^{\mathrm{a}, \mathrm{b}}$ & Compd & $t_{\mathrm{R}}(\mathrm{min})$ & Eluent $^{\mathrm{a}, \mathrm{b}}$ \\
\hline$(5 S, 6 R)-\mathbf{1 0 a}$ & 17.24 & $98 / 2$ & $(5 R, 6 R)-\mathbf{1 1 f}$ & $6.36^{\mathrm{c}}$ & $90 / 10$ \\
$(5 R, 6 S)-\mathbf{1 0 a}$ & $13.82^{\mathrm{c}}$ & $98 / 2$ & $(5 S, 6 S)-\mathbf{1 1 f}$ & 6.71 & $90 / 10$ \\
$(5 S, 6 R)-\mathbf{1 1 a}$ & $5.42^{\mathrm{c}}$ & $98 / 2$ & $(5 R, 6 R)-\mathbf{1 0 g}$ & $\mathrm{d}$ & - \\
$(5 R, 6 S)-\mathbf{1 1 a}$ & 6.20 & $98 / 2$ & $(5 S, 6 S)-\mathbf{1 0 g}$ & $\mathrm{d}$ & - \\
$(5 R, 6 S)-\mathbf{1 0 d}$ & $10.90^{\mathrm{c}}$ & $95 / 5$ & $(5 R, 6 R)-\mathbf{1 1 g}$ & $7.14^{\mathrm{c}}$ & $98 / 2$ \\
$(5 S, 6 R)-\mathbf{1 0 d}$ & 11.65 & $95 / 5$ & $(5 S, 6 S)-\mathbf{1 1}$ & 8.67 & $98 / 2$ \\
$(5 S, 6 R)-\mathbf{1 1 d}$ & $16.84^{\mathrm{c}}$ & $98 / 2$ & $(5 R, 6 R)-\mathbf{1 0 h}$ & 24.15 & $98 / 2$ \\
$(5 R, 6 S)-\mathbf{1 1 d}$ & 17.53 & $98 / 2$ & $(5 S, 6 S)-\mathbf{1 0 h}$ & $25.45^{\mathrm{c}}$ & $98 / 2$ \\
$(5 R, 6 R)-\mathbf{1 0 e}$ & 20.78 & $98 / 2$ & $(5 R, 6 R)-\mathbf{1 1 h}$ & $10.29^{\mathrm{c}}$ & $98 / 2$ \\
$(5 S, 6 S)-\mathbf{1 0}$ & $27.61^{\mathrm{c}}$ & $98 / 2$ & $(5 S, 6 S)-\mathbf{1 1}$ & 10.95 & $98 / 2$ \\
$(5 R, 6 R)-\mathbf{1 1 e}$ & $8.15^{\mathrm{c}}$ & $98 / 2$ & $(5 R, 6 R)-\mathbf{1 0 i}$ & 49.55 & $98 / 2^{\mathrm{e}}$ \\
$(5 S, 6 S)-\mathbf{1 1 e}$ & 8.63 & $98 / 2$ & $(5 S, 6 S)-\mathbf{1 0 i}$ & $53.46^{\mathrm{c}}$ & $98 / 2^{\mathrm{e}}$ \\
$(5 R, 6 R)-\mathbf{1 0 f}$ & 8.67 & $90 / 10$ & $(5 R, 6 R)-\mathbf{1 1 i}$ & $23.51^{\mathrm{c}}$ & $98 / 2^{\mathrm{e}}$ \\
$(5 S, 6 S)-\mathbf{1 0 f}$ & $10.89^{\mathrm{c}}$ & $90 / 10$ & $(5 S, 6 S)-\mathbf{1 1 i}$ & 26.18 & $98 / 2^{\mathrm{e}}$ \\
\hline
\end{tabular}

${ }^{a} n$-Hexane/i-propanol.

${ }^{\mathrm{b}}$ Flow $1 \mathrm{~mL} / \mathrm{min}$.

${ }^{\mathrm{c}}$ Major enantiomer.

${ }^{\mathrm{d}}$ Determined as ester

${ }^{\mathrm{e}}$ Flow $0.6 \mathrm{~mL} / \mathrm{min}$.
The configuration of the products was assigned by analogy with resolution of compound ( \pm )-12, which was resolved using the same lipase (lipase PS on Celite; Scheme 1). ${ }^{21}$ In the cases of compounds $10 \mathrm{~h}$ and $11 \mathrm{~h}$, which were poorly resolved, configuration was tentatively assigned since such compounds showed the same elution order and Cotton effect of compounds 10i and 11i.

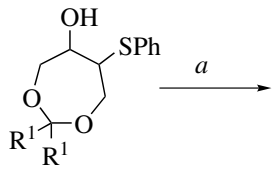

(士)-anti-12

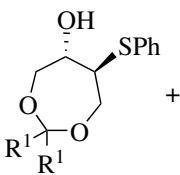

$(S, S)-\mathbf{1 3}$

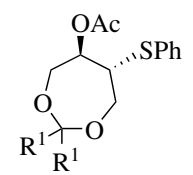

$(R, R)-14$
Scheme 1. Reagents and conditions: (a) PS-on-Celite, $i-\operatorname{Pr}_{2} \mathrm{O}$, vinyl acetate, $30^{\circ} \mathrm{C}$.

When considering substrates having the dimethyl acetal group, the best resolution was obtained with $\mathrm{R}=\mathrm{SePh}$. With the less bulky azido group, the resolution was slightly less good. Despite the bulkiness of the benzyloxy substituent, compound $( \pm)-\mathbf{8 h}$ showed very poor selectivity, probably because of the free rotation of such a group. The low enantiopreference observed is in agreement with the fact that the $\mathrm{CH}_{2}$ group can also be fitted as a medium sized substituent into the stereoselective pocket of the enzyme, whereas the dimethyl acetal group acts as a large substituent (Fig. 4).

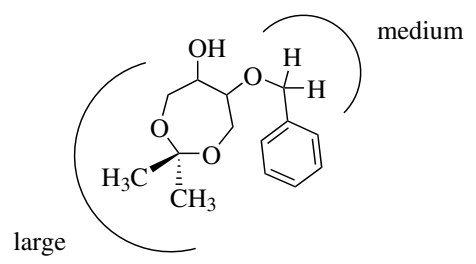

(士)-anti-8h

Figure 4. Size of substituents.

Following Ema's model, ${ }^{22}$ the medium group should be fitted into the stereoselectivity pocket, whereas the large one is directed towards the external solvent (Fig. 5). On the grounds of simple computer molecular models, we can reasonably estimate that the size of the dimethyl group is able to produce an effective steric hindrance for the penetration

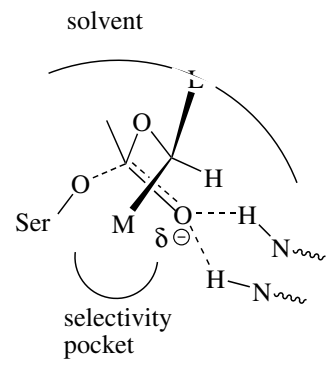

Figure 5. Ema's model. 
of the species into the enzyme pocket, then acting as a large substituent too. 23

In compounds $\mathbf{8 a}$ and $\mathbf{8 e}$, the azido and the phenylseleno groups must act as large groups. Indeed, it is not possible to find a less hindered conformation. For these substrates, the portion of the molecule containing the dimethylacetal group was forced to be the medium one, causing a low reaction rate because of the steric repulsion.

Indeed, when the dimethyl acetal group was removed the reaction rates were high giving excellent enantiopreference for $\mathrm{R}=\mathrm{SePh}, \mathrm{OCH}_{2} \mathrm{Ph}$ and $\mathrm{N}_{3}$. The difference between $\mathrm{N}_{3}$ and I could be ascribed to the higher polarizability of the iodine atom.

Finally, we carried out a simple manipulation. Compound 10i was oxidized using stabilized 2-iodoxybenzoic acid ${ }^{24}$ to give a protected form of $(S)$-erythrulose (Scheme 2 ).

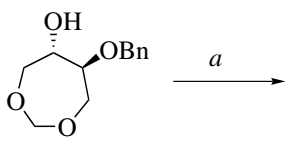

$10 \mathrm{i}$

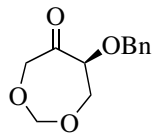

15
Scheme 2. Reagents and conditions: (a) 2-iodoxybenzoic acid, ethyl acetate, $75^{\circ} \mathrm{C}, 91 \%$.

Compound $\mathbf{1 5}$ is a valuable building block that is currently used in our laboratory as a starting material for stereoselective transformations.

\section{Conclusion}

In conclusion, useful enantiopure building blocks have been obtained by enzymatic resolution of racemic anti-6substituted 1,3-dioxepan-5-ols. Excellent resolutions were obtained with large $\mathrm{R}$ substituent ( $\mathrm{PhSe}$ ). With the smaller $\mathrm{N}_{3}$ substituent, excellent resolution was observed with $\mathrm{R}^{1}=\mathrm{H}$. When $\mathrm{R}^{1}=\mathrm{Me}$ the enantiopreference was lower because of the steric hindrance caused by the portion of the molecule containing the dimethyl acetal group. The iodine substituent $(\mathrm{R}=\mathrm{I})$ did not give excellent results even with the unsubstituted acetal. Finally, the benzyloxy substituent may also act as a medium substituent giving a low enantiopreference when the dimethylacetal group was present, whereas it acts as a large substituent when the dimethyl group was absent giving an excellent resolution. These compounds are precursors of compounds 1-3, but other simple manipulations can be carried out. For example, oxidation of compound $\mathbf{1 0 i}$ gave a protected form of $(S)$ erythrulose.

\section{Experimental}

\subsection{General}

The NMR spectra were recorded on a Bruker AC-E series $250 \mathrm{MHz}$ or $300 \mathrm{MHz}$ spectrometer using $\mathrm{CDCl}_{3}$ as solvent. FT-IR spectra were registered with a Shimadzu FTIR
8300 infrared spectrophotometer. Carbon and hydrogen contents were determined by combustion analysis in a Fisons EA 1108 elemental analyzer. Optical rotations were measured in chloroform on a Jasco P1010 polarimeter. Chiral HPLC was performed using a Shimadzu LC10AD pump with a SPD-M10A UV detector and Daicel column (OD-H). Compounds $( \pm)$-anti-8a-i were prepared starting from the proper cyclic epoxide. 2,2-Dimethyl-1,3dioxepan-5,6-oxirane and 1,3-dioxepan-5,6-oxirane were obtained by epoxidation $^{6 a}$ of 2,2-dimethyl-1,3-dioxep-5-en or dimethyl-1,3-dioxep-5-en. Epoxide ring opening was carried out with $\mathrm{NaN}_{3}, \mathrm{NaSePh}$, NaI or $\mathrm{BnOH}^{25}$ Compounds $( \pm)$-anti-8b-c and $( \pm)$-anti-9 were prepared after reduction of the azido group with $\mathrm{Ni}-\mathrm{Raney}$ and protection.

\subsection{Enzymatic resolution procedure}

To a solution of alcohol $( \pm)-8(0.4 \mathrm{mmol})$ in toluene $(0.9 \mathrm{~mL})$ at $30^{\circ} \mathrm{C}$, vinyl acetate $(1.2 \mathrm{mmol})$ and PS-C II lipase $(50 \mathrm{mg})$ were added. The reaction was monitored by HPLC, then taken up with diethyl ether, filtered and the solvent removed under reduced pressure. The residue was checked by NMR, then purified by silica gel chromatography using light petroleum/ethyl acetate as eluent.

4.2.1. (5R,6S)-6-Azido-2,2-dimethyl-1,3-dioxepan-5-ol 10a. $[\alpha]_{\mathrm{D}}^{27}=+91.9 \quad\left(c \quad 0.40, \mathrm{CHCl}_{3}\right) ;>99 \%$ ee. ${ }^{1} \mathrm{H}$ NMR (250 MHz): $\delta 1.34$ (s, 6H), 2.87 (br s, $1 \mathrm{H}, \mathrm{OH}), 3.36-3.43$ $(\mathrm{m}, 1 \mathrm{H}), 3.55-3.74(\mathrm{~m}, 3 \mathrm{H}), 3.80-3.90(\mathrm{~m}, 2 \mathrm{H}) ;{ }^{13} \mathrm{C}$ NMR (62.5 MHz): $\delta$ 24.2, 24.6, 58.9, 61.4, 64.3, 71.2, 101.9. IR (liquid film) 3423, 2108, $1452,1375 \mathrm{~cm}^{-1}$. Anal. Calcd for $\mathrm{C}_{7} \mathrm{H}_{13} \mathrm{~N}_{3} \mathrm{O}_{3}$ : C, 44.91; $\mathrm{H}, 7.00$. Found: $\mathrm{C}$, 44.78; H, 7.13.

4.2.2. (5S,6R)-6-Azido-2,2-dimethyl-1,3-dioxepan-5-yl acetate 11a. $[\alpha]_{\mathrm{D}}^{22}=-43.3\left(c \quad 0.49, \mathrm{CHCl}_{3}\right) ; 93 \%$ ee. ${ }^{1} \mathrm{H}$ NMR (250 MHz): $\delta 1.34(\mathrm{~s}, 6 \mathrm{H}), 2.10(\mathrm{~s}, 3 \mathrm{H}), 3.49-3.54$ $(\mathrm{m}, 1 \mathrm{H}), 3.63-3.85(\mathrm{~m}, 4 \mathrm{H}), 4.76-4.82(\mathrm{~m}, 1 \mathrm{H}) ;{ }^{13} \mathrm{C}$ NMR (62.5 MHz): $\delta$ 20.9, 24.1, 24.3, 59.6, 59.7, 62.4, 73.6, 101.7, 169.7. IR (liquid film) 2112, 1745, 1454, $1372 \mathrm{~cm}^{-1}$. Anal. Calcd for $\mathrm{C}_{9} \mathrm{H}_{15} \mathrm{~N}_{3} \mathrm{O}_{4}: \mathrm{C}, 47.16 ; \mathrm{H}$, 6.60. Found: C, 47.32; H, 6.71.

\subsection{3. ( \pm )-anti-Benzyl 6-hydroxy-2,2-dimethyl-1,3-dioxepan-} 5-yl carbamate $8 b$. To a solution of $( \pm)$-anti-6-amino-2,2dimethyl-1,3-dioxepan-5-ol (98 mg, $0.608 \mathrm{mmol})$ in methanol (1.2 mL), $\mathrm{Na}_{2} \mathrm{CO}_{3}(0.73 \mathrm{mmol})$ was added. The mixture was cooled at $0{ }^{\circ} \mathrm{C}$, then benzyl chloroformate $(85 \mu \mathrm{L}$, $0.73 \mathrm{mmol}$ ) was slowly added. The mixture was stirred for $2 \mathrm{~h}$ at room temperature, then filtered and the solvent removed under reduced pressure. The residue was purified by column chromatography using light petroleum/ethyl acetate $2 / 1$ as eluent. Yield $65 \%$. ${ }^{1} \mathrm{H}$ NMR (250 MHz): $\delta$ $1.33(\mathrm{~s}, 3 \mathrm{H}), 1.36(\mathrm{~s}, 3 \mathrm{H}), 3.28(\mathrm{~s}, 1 \mathrm{H}, \mathrm{OH}), 3.49$ (ddd, $J=12.7,2.2$ and $2.2 \mathrm{~Hz}, 1 \mathrm{H}), 3.51-3.81(\mathrm{~m}, 4 \mathrm{H}), 4.03(\mathrm{~d}$, $J=12.7 \mathrm{~Hz}, 1 \mathrm{H}), 5.11-5.17(\mathrm{~m}, 2 \mathrm{H}), 5.55(\mathrm{~d}, J=7.4 \mathrm{~Hz}$, $1 \mathrm{H}), 7.33-7.40(\mathrm{~m}, 5 \mathrm{H})$.

4.2.4. (士)-anti-N-(6-Hydroxy-2,2-dimethyl-1,3-dioxepan-5yl)-benzamide 8c. A solution of benzoyl chloride $(109 \mu \mathrm{L}, 0.943 \mathrm{mmol})$ in dichloromethane $(1 \mathrm{~mL})$ was 
slowly added to a solution of $( \pm)$-anti-6-amino-2,2-dimethyl-1,3-dioxepan-5-ol (152 mg, $0.943 \mathrm{mmol})$ and $\mathrm{NaH}$ $\mathrm{CO}_{3}(1.13 \mathrm{mmol})$ in water $(1.6 \mathrm{~mL})$. The mixture was stirred for $2 \mathrm{~h}$ at room temperature, then water was added $(15 \mathrm{~mL})$ and the solution extracted with dichloromethane $(3 \times 15 \mathrm{~mL})$ and brine $(1 \times 15 \mathrm{~mL})$. The organic solvent was removed under reduced pressure to give pure compound $8 \mathrm{c}(80 \%)$. Mp $146{ }^{\circ} \mathrm{C} .{ }^{1} \mathrm{H}$ NMR $(300 \mathrm{MHz}): \delta 1.35$ $(\mathrm{s}, 3 \mathrm{H}), 1.36(\mathrm{~s}, 3 \mathrm{H}), 3.54(\mathrm{dd}, J=12.4$ and $3.9 \mathrm{~Hz}, 1 \mathrm{H})$, $3.64(\mathrm{dd}, J=13.1$ and $3.9 \mathrm{~Hz}, 1 \mathrm{H}), 3.76-3.81(\mathrm{~m}, 2 \mathrm{H})$, 4.11-4.16 (m, 2H), $4.19(\mathrm{br} \mathrm{s}, 1 \mathrm{H}, \mathrm{OH}), 6.98(\mathrm{~d}$, $J=7.7 \mathrm{~Hz}, 1 \mathrm{H}), 7.35-7.51(\mathrm{~m}, 3 \mathrm{H}), 7.74-7.77(\mathrm{~m}, 2 \mathrm{H})$; ${ }^{13} \mathrm{C}$ NMR $(75 \mathrm{MHz}): \delta$ 24.8, 25.2, 54.8, 59.7, 62.3, 70.6, $102.3,127.4,128.9,132.1,134.5,167.7$. IR (nujol) 3382, 3289, 1638, 1626, $1554 \mathrm{~cm}^{-1}$. Anal. Calcd for $\mathrm{C}_{14} \mathrm{H}_{19} \mathrm{NO}_{4}$ : C, 63.38; H, 7.22. Found: C, 63.51; H, 7.33.

4.2.5. (5R,6S)-6-Azido-1,3-dioxepan-5-ol 10d. Mp 44$46{ }^{\circ} \mathrm{C} ;[\alpha]_{\mathrm{D}}^{23}=+50.8\left(\right.$ c $\left.0.48, \mathrm{CHCl}_{3}\right) ;>99 \%$ ee. ${ }^{1} \mathrm{H} \mathrm{NMR}$ (250 MHz): $\delta 2.98$ (br s, $1 \mathrm{H}, \mathrm{OH}), 3.48-3.54(\mathrm{~m}, 1 \mathrm{H})$, $3.67-3.90(\mathrm{~m}, 5 \mathrm{H}), 4.71(\mathrm{~d}, J=4.5 \mathrm{~Hz}, 1 \mathrm{H}), 4.74(\mathrm{~d}$, $J=4.5 \mathrm{~Hz}, 1 \mathrm{H}) ;{ }^{13} \mathrm{C} \mathrm{NMR}(62.5 \mathrm{MHz}): \delta$ 63.7, 64.8, 65.7, 71.8, 94.1. IR (liquid film) 3432, 2104, $1457 \mathrm{~cm}^{-1}$. Anal. Calcd for $\mathrm{C}_{5} \mathrm{H}_{9} \mathrm{~N}_{3} \mathrm{O}_{3}$ : C, 37.74; $\mathrm{H}$, 5.70. Found: C, 37.99; H, 5.60.

4.2.6. (5S,6R)-6-Azido-1,3-dioxepan-5-yl acetate 11d. $[\alpha]_{\mathrm{D}}^{23}=-40.35 \quad\left(c \quad 0.49, \mathrm{CHCl}_{3}\right) ; \quad 96 \%$ ee. ${ }^{1} \mathrm{H} \quad \mathrm{NMR}$ $(250 \mathrm{MHz}): \delta 2.11(\mathrm{~s}, 3 \mathrm{H}), 3.63(\mathrm{ddd}, J=6.1,6.1$ and $2.9 \mathrm{~Hz}, 1 \mathrm{H}), 3.71-3.82(\mathrm{~m}, 2 \mathrm{H}), 3.84(\mathrm{dd}, J=13.7$ and $3.0 \mathrm{~Hz}, 1 \mathrm{H}), 3.93(\mathrm{dd}, J=12.5$ and $3.0 \mathrm{~Hz}, 1 \mathrm{H}), 4.73(\mathrm{~d}$, $J=4.7 \mathrm{~Hz}, 1 \mathrm{H}), 4.76(\mathrm{~d}, J=4.7 \mathrm{~Hz}, 1 \mathrm{H}), 4.87$ (ddd, $J=6.0,6.0$ and $3.1 \mathrm{~Hz}, 1 \mathrm{H}) ;{ }^{13} \mathrm{C} \mathrm{NMR}(62.5 \mathrm{MHz}): \delta$ $20.8,62.8,64.0,64.5,73.9,94.4,169.7$. IR (liquid film) 2107, 1735, $1374 \mathrm{~cm}^{-1}$. Anal. Calcd for $\mathrm{C}_{7} \mathrm{H}_{11} \mathrm{~N}_{3} \mathrm{O}_{4}: \mathrm{C}$, 41.79; H, 5.51. Found: C, 41.98; H, 5.62.

4.2.7. (5S,6S)-2,2-Dimethyl-6-(phenylseleno)-1,3-dioxepan5-ol 10e. $[\alpha]_{\mathrm{D}}^{26}=+17.4\left(c \quad 1.02, \mathrm{CHCl}_{3}\right) ;>99 \%$ ee. ${ }^{1} \mathrm{H}$ NMR (300 MHz): $\delta 1.36(\mathrm{~s}, 6 \mathrm{H}), 2.81(\mathrm{~d}, J=5.4 \mathrm{~Hz}, 1 \mathrm{H}$, $\mathrm{OH}), 3.19-3.23(\mathrm{~m}, 1 \mathrm{H}), 3.61-3.71(\mathrm{~m}, 2 \mathrm{H}), 3.83(\mathrm{dd}$, $J=12.8$ and $6.1 \mathrm{~Hz}, 1 \mathrm{H}), 4.08-4.16(\mathrm{~m}, 2 \mathrm{H}), 7.27-7.31$ $(\mathrm{m}, 3 \mathrm{H}), 7.56-7.60(\mathrm{~m}, 2 \mathrm{H}) ;{ }^{13} \mathrm{C} \mathrm{NMR}(75 \mathrm{MHz}): \delta 24.5$, $24.7,51.7,61.2,62.8,71.6,101.6,127.7,128.3,129.2$, 134.3. IR (liquid film) $3420,1580,1477,1437,1373 \mathrm{~cm}^{-1}$. Anal. Calcd for $\mathrm{C}_{13} \mathrm{H}_{18} \mathrm{O}_{3} \mathrm{Se}$ : C, 51.83; H, 6.02. Found: C, 51.70; H, 6.20.

4.2.8. (5R,6R)-2,2-Dimethyl-6-(phenylseleno)-1,3-dioxepan5-yl acetate 11e. $[\alpha]_{\mathrm{D}}^{26}=-29.8\left(c 1.47, \mathrm{CHCl}_{3}\right) ;>99 \%$ ee. ${ }^{1} \mathrm{H}$ NMR (250 MHz): $\delta$; ${ }^{13} \mathrm{C}$ NMR $(62.5 \mathrm{MHz}): \delta 1.35(\mathrm{~s}$, $6 \mathrm{H}), 2.07(\mathrm{~s}, 3 \mathrm{H}), 3.25-3.35(\mathrm{~m}, 1 \mathrm{H}), 3.69-3.84(\mathrm{~m}, 2 \mathrm{H})$, 4.07-4.17 (m, 2H), 4.90-4.96 (m, 1H), 7.26-7.31 (m, 3H), 7.57-7.63 (m, 2H). IR (liquid film) 1738, 1580, 1480, 1437, $1371 \mathrm{~cm}^{-1}$. Anal. Calcd for $\mathrm{C}_{15} \mathrm{H}_{20} \mathrm{O}_{4} \mathrm{Se}$ : C, 52.48; H, 5.87. Found: C, 52.60; H, 5.99.

4.2.9. (5S,6S)-6-(Phenylseleno)-1,3-dioxepan-5-ol 10f. $\mathrm{Mp}$ 54-55 ${ }^{\circ} \mathrm{C}$. $[\alpha]_{\mathrm{D}}^{26}=+4.0\left(c \quad 0.95, \mathrm{CHCl}_{3}\right) ;>99 \%$ ee. ${ }^{1} \mathrm{H}$ NMR (300 MHz): $\delta 3.10$ (br s, $1 \mathrm{H}, \mathrm{OH}), 3.42-3.52(\mathrm{~m}$, $1 \mathrm{H}), 3.97-4.03(\mathrm{~m}, 2 \mathrm{H}), 4.14(\mathrm{dd}, J=12.5$ and $6.3 \mathrm{~Hz}$, $1 \mathrm{H}), 4.33-4.41$ (m, 2H), $4.97(\mathrm{~d}, J=4.5 \mathrm{~Hz}, 1 \mathrm{H}), 5.00(\mathrm{~d}$,
$J=4.5 \mathrm{~Hz}, 1 \mathrm{H}), 7.50-7.55(\mathrm{~m}, 3 \mathrm{H}), 7.78-7.81(\mathrm{~m}, 2 \mathrm{H})$ ${ }^{13} \mathrm{C}$ NMR (75 MHz): $\delta 51.6,65.8,66.7,72.0,94.1,127.6$, 128.0, 129.3, 134.6. IR (nujol) $3345 \mathrm{~cm}^{-1}$. Anal. Calcd for $\mathrm{C}_{11} \mathrm{H}_{14} \mathrm{O}_{3} \mathrm{Se}$ : C, 48.36; H, 5.17. Found: C, 48.52; H, 5.30 .

4.2.10. (5R,6R)-6-(Phenylseleno)-1,3-dioxepan-5-yl acetate 11f. $[\alpha]_{\mathrm{D}}^{25}=-15.0\left(c 1.06, \mathrm{CHCl}_{3}\right) ;>99 \%$ ee. ${ }^{1} \mathrm{H} \mathrm{NMR}$ (300 MHz): $\delta 2.00(\mathrm{~s}, 3 \mathrm{H}), 3.27-3.31(\mathrm{~m}, 1 \mathrm{H}), 3.77-3.84$ $(\mathrm{m}, 2 \mathrm{H}), 4.02(\mathrm{dd}, J=12.6$ and $2.3 \mathrm{~Hz}, 1 \mathrm{H}), 4.14(\mathrm{dd}$, $J=12.3$ and $2.4 \mathrm{~Hz}, 1 \mathrm{H}), 4.66(\mathrm{~d}, J=4.5 \mathrm{~Hz}, 1 \mathrm{H}), 4.72$ $(\mathrm{d}, J=4.5 \mathrm{~Hz}, 1 \mathrm{H}), 4.92-4.97(\mathrm{~m}, 1 \mathrm{H}), 7.19-7.25(\mathrm{~m}$, $3 \mathrm{H}), 7.50-7.53(\mathrm{~m}, 2 \mathrm{H}) ;{ }^{13} \mathrm{C}$ NMR $(75 \mathrm{MHz}): \delta 21.0$, $48.0,65.7,65.8,74.3,94.3,127.8,127.9,129.1,134.5$, 169.9. IR (liquid film) $1733,1578 \mathrm{~cm}^{-1}$. Anal. Calcd for $\mathrm{C}_{13} \mathrm{H}_{16} \mathrm{O}_{4} \mathrm{Se}: \mathrm{C}, 49.53$; H, 5.12. Found: C, 49.21; H, 5.01.

4.2.11. (5S,6S)-6-Iodo-1,3-dioxepan-5-ol 10g. $[\alpha]_{\mathrm{D}}^{24}=$ +14.4 ( $\left.c 0.55, \mathrm{CHCl}_{3}\right) ; 70 \%$ ee. ${ }^{1} \mathrm{H}$ NMR $(250 \mathrm{MHz}): \delta$ 3.26 (br s, $1 \mathrm{H}, \mathrm{OH}), 3.66-4.13(\mathrm{~m}, 6 \mathrm{H}), 4.76-4.78(\mathrm{~m}$, $2 \mathrm{H}) ;{ }^{13} \mathrm{C}$ NMR $(62.5 \mathrm{MHz}): \delta 36.5,66.0,67.0,75.5,77.2$, 94.0. IR (liquid film) $3425,1452 \mathrm{~cm}^{-1}$. Anal. Calcd for $\mathrm{C}_{7} \mathrm{H}_{13} \mathrm{IO}_{3}$ : C, 30.90; H, 4.82. Found: C, 30.99; H, 4.90.

4.2.12. (5R,6R)-6-Iodo-1,3-dioxepan-5-yl acetate 11g. $[\alpha]_{\mathrm{D}}^{23}=-41.7 \quad\left(c \quad 0.79, \quad \mathrm{CHCl}_{3}\right) ; 90 \%$ ee. ${ }^{1} \mathrm{H} \quad \mathrm{NMR}$ (250 MHz): $\delta 2.12(\mathrm{~s}, 3 \mathrm{H}), 3.72(\mathrm{dd}, J=12.2$ and $7.0 \mathrm{~Hz}$, $1 \mathrm{H}), 3.85(\mathrm{dd}, J=12.5$ and $8.0 \mathrm{~Hz}, 1 \mathrm{H}), 3.97(\mathrm{dd}$, $J=12.2$ and $3.1 \mathrm{~Hz}, 1 \mathrm{H}), 4.03-4.15(\mathrm{~m}, 2 \mathrm{H}), 4.79(\mathrm{~d}$, $J=4.5 \mathrm{~Hz}, 1 \mathrm{H}), 4.82(\mathrm{~d}, J=4.5 \mathrm{~Hz}, 1 \mathrm{H}), 5.09-5.15(\mathrm{~m}$, $1 \mathrm{H}) ;{ }^{13} \mathrm{C}$ NMR $(62.5 \mathrm{MHz}): \delta 21.3,30.6,65.4,67.7,77.3$, 94.6, 170.0. IR (liquid film) $1735,1369 \mathrm{~cm}^{-1}$. Anal. Calcd for $\mathrm{C}_{9} \mathrm{H}_{15} \mathrm{IO}_{4}$ : C, 34.41; H, 4.81. Found: C, 34.61; H, 4.99.

4.2.13. 6-(Benzyloxy)-2,2-dimethyl-1,3-dioxepan-5-ol 10h. $[\alpha]_{\mathrm{D}}^{25}=+2.0 \quad\left(c \quad 1.26, \quad \mathrm{CHCl}_{3}\right) ; 14 \%$ ee. ${ }^{1} \mathrm{H} \quad \mathrm{NMR}$ $(300 \mathrm{MHz}): \delta 1.29(\mathrm{~s}, 3 \mathrm{H}), 1.36(\mathrm{~s}, 3 \mathrm{H}), 2.78(\mathrm{br} \mathrm{s}, 1 \mathrm{H}$, $\mathrm{OH}), 3.37-3.48(\mathrm{~m}, 2 \mathrm{H}), 3.57(\mathrm{dd}, J=11.5$ and $4.3 \mathrm{~Hz}$, $1 \mathrm{H}), 3.65(\mathrm{dd}, J=7.6$ and $7.6 \mathrm{~Hz}, 1 \mathrm{H}), 3.87(\mathrm{dd}$, $J=6.6 \mathrm{~Hz}, 1 \mathrm{H}), 4.16(\mathrm{ddd}, J=6.6,6.6$ and $6.6 \mathrm{~Hz}, 1 \mathrm{H})$, $4.57(\mathrm{~d}, J=11.9 \mathrm{~Hz}, 1 \mathrm{H}), 4.63(\mathrm{~d}, J=11.9 \mathrm{~Hz}, 1 \mathrm{H})$, 7.14-7.27 (m, 5H); ${ }^{13} \mathrm{C}$ NMR (75 MHz): $\delta$ 25.1, 26.1, $61.4,65.3,72.4,76.3,79.2,109.0,127.4,127.6,128.1$, 138.0. IR (liquid film) $3457,1454,1380,1370 \mathrm{~cm}^{-1}$. Anal. Calcd for $\mathrm{C}_{14} \mathrm{H}_{20} \mathrm{O}_{4}$ : C, 66.65; H, 7.99. Found: C, 66.80; H, 8.14.

4.2.14. 6-(Benzyloxy)-2,2-dimethyl-1,3-dioxepan-5-yl acetate 11h. $[\alpha]_{\mathrm{D}}^{26}=-2.6\left(c \quad 0.69, \mathrm{CHCl}_{3}\right) ; 14 \%$ ee. ${ }^{1} \mathrm{H}$ NMR (300 MHz): $\delta 1.29(\mathrm{~s}, 3 \mathrm{H}), 1.35(\mathrm{~s}, 3 \mathrm{H}), 1.97(\mathrm{~s}$, $3 \mathrm{H}), 3.47-3.54(\mathrm{~m}, 2 \mathrm{H}), 3.60-3.68(\mathrm{~m}, 1 \mathrm{H}), 3.73(\mathrm{dd}$, $J=8.3$ and $7.1 \mathrm{~Hz}, 1 \mathrm{H}), 3.93(\mathrm{dd}, J=8.3$ and $6.6 \mathrm{~Hz}$, 1H), 4.19-4.27 (m, 1H), $4.62(\mathrm{~d}, J=11.8 \mathrm{~Hz}, 1 \mathrm{H}), 4.69$ $(\mathrm{d}, J=11.8 \mathrm{~Hz}, 1 \mathrm{H}), 7.21-7.29 \quad(\mathrm{~m}, 5 \mathrm{H}) ;{ }^{13} \mathrm{C}$ NMR $(75 \mathrm{MHz}): \delta 20.9,25.3,27.3,63.6,65.5,72.9,76.0,76.8$, 109.5, 127.8, 127.9, 128.4, 138.1, 170.8. IR (liquid film) $1742 \mathrm{~cm}^{-1}$. Anal. Calcd for $\mathrm{C}_{16} \mathrm{H}_{22} \mathrm{O}_{5}: \mathrm{C}, 65.29 ; \mathrm{H}, 7.53$. Found: C, 65.50; H, 7.67.

4.2.15. (5S,6S)-6-(Benzyloxy)-1,3-dioxepan-5-ol 10i. $[\alpha]_{\mathrm{D}}^{30}=+29.0 \quad\left(c \quad 0.82, \mathrm{CHCl}_{3}\right) ;>99 \%$ ee. ${ }^{1} \mathrm{H} \quad \mathrm{NMR}$ (300 MHz): $\delta 2.70(\mathrm{br} s, 1 \mathrm{H}, \mathrm{OH}), 3.26-3.29(\mathrm{~m}, 1 \mathrm{H})$, 
3.48-3.61 (m, 4H), $3.76(\mathrm{dd}, J=11.6$ and $1.1 \mathrm{~Hz}, 1 \mathrm{H}), 4.38$ $(\mathrm{d}, J=12.0 \mathrm{~Hz}, 1 \mathrm{H}), 4.45(\mathrm{~d}, J=12.0 \mathrm{~Hz}, 1 \mathrm{H}), 4.52-4.56$ (m, 2H), 7.06-7.15 (m, 5H); ${ }^{13} \mathrm{C}$ NMR (75 MHz): $\delta 64.4$, 65.6, 71.4, 71.5, 80.3, 94.5, 127.7, 127.8, 128.4, 137.9. IR (liquid film) $3445,1454 \mathrm{~cm}^{-1}$. Anal. Calcd for $\mathrm{C}_{12} \mathrm{H}_{16} \mathrm{O}_{4}$ : C, 64.27; H, 7.19. Found: C, 64.42; H, 7.27.

4.2.16. (5R,6R)-6-(Benzyloxy)-1,3-dioxepan-5-yl acetate 11i. $[\alpha]_{\mathrm{D}}^{3 \mathrm{1}}=-11.7\left(\right.$ c $\left.0.88, \mathrm{CHCl}_{3}\right) ;>99 \%$ ee. ${ }^{1} \mathrm{H} \mathrm{NMR}$ (300 MHz): $\delta 2.00(\mathrm{~s}, 3 \mathrm{H}), 3.42-3.47(\mathrm{~m}, 1 \mathrm{H}), 3.74-3.80$ $(\mathrm{m}, 3 \mathrm{H}), 3.89(\mathrm{dd}, J=12.9$ and $2.2 \mathrm{~Hz}, 1 \mathrm{H}), 4.60(\mathrm{~s}, 2 \mathrm{H})$, $4.69(\mathrm{~d}, J=4.6 \mathrm{~Hz}, 1 \mathrm{H}), 4.72(\mathrm{~d}, J=4.6 \mathrm{~Hz}, 1 \mathrm{H}), 4.90$ $4.94(\mathrm{~m}, 1 \mathrm{H}), 7.21-7.28(\mathrm{~m}, 5 \mathrm{H}) ;{ }^{13} \mathrm{C}$ NMR $(75 \mathrm{MHz}): \delta$ $20.11,63.6,63.7,70.5,72.1,76.8,93.7,126.9,127.4$, 136.9, 169.1. IR (liquid film) 1739, 1454, 1371, $1238 \mathrm{~cm}^{-1}$. Anal. Calcd for $\mathrm{C}_{14} \mathrm{H}_{18} \mathrm{O}_{5}: \mathrm{C}, 63.15 ; \mathrm{H}, 6.81$. Found: C, 63.25; H, 6.98 .

4.2.17. ( \pm )-anti-6-Acetamido-2,2-dimethyl-1,3-dioxepan-5-yl acetate 9. To a solution of ( \pm )-anti-6-amino-2,2-dimethyl-1,3-dioxepan-5-ol (125 mg, $0.775 \mathrm{mmol})$ in $\mathrm{N}, \mathrm{N}$ dimethylformamide $(0.5 \mathrm{~mL}), \mathrm{Na}_{2} \mathrm{CO}_{3}(1.55 \mathrm{mmol})$ and acetic anhydride $(1 \mathrm{~mL})$ were added. The mixture was stirred for $2 \mathrm{~h}$ at room temperature, then water $(20 \mathrm{~mL})$ was added and the mixture extracted with ethyl acetate $(3 \times 15 \mathrm{~mL})$ and brine $(1 \times 15 \mathrm{~mL})$. The solvent was removed under reduced pressure and the residue purified by washing with diethyl ether. Yield $90 \%$. Mp $123{ }^{\circ} \mathrm{C} .{ }^{1} \mathrm{H}$ NMR $(300 \mathrm{MHz}): \delta 1.36(\mathrm{~s}, 3 \mathrm{H}), 1.38(\mathrm{~s}, 3 \mathrm{H}), 2.03(\mathrm{~s}$, $3 \mathrm{H}), 2.11(\mathrm{~s}, 3 \mathrm{H}), 3.49(\mathrm{dd}, J=12.6$ and $4.2 \mathrm{~Hz}, 1 \mathrm{H})$, $3.72(\mathrm{~d}, J=13.5 \mathrm{~Hz}, 1 \mathrm{H}), 3.84(\mathrm{dd}, J=13.5$ and $3.9 \mathrm{~Hz}$, $1 \mathrm{H}), 4.00-4.08(\mathrm{~m}, 2 \mathrm{H}), 4.72-4.76(\mathrm{~m}, 1 \mathrm{H}), 6.04(\mathrm{~d}$, $J=6.9 \mathrm{~Hz}, 1 \mathrm{H}) ;{ }^{13} \mathrm{C}$ NMR $(75 \mathrm{MHz}): \delta 21.5,23.7,24.6$, $25.2,51.9,59.8,60.4,72.8,102.3,162.7,169.8$. IR (nujol) $3231, \quad 3068,1736,1644 \mathrm{~cm}^{-1}$. Anal. Calcd for $\mathrm{C}_{11} \mathrm{H}_{19} \mathrm{NO}_{5}$ : C, 53.87; H, 7.81. Found: C, 53.60; H, 7.98.

4.2.18. (S)-6-Benzyloxy-1,3-dioxepan-5-one 15. To a solution of compound $10 \mathbf{i}(300 \mathrm{mg}, 1.34 \mathrm{mmol})$ in ethyl acetate $(9.5 \mathrm{~mL})$ was added 2-iodoxybenzoic acid (1.2 equiv, $1.0 \mathrm{~g}$ ) and the mixture heated at $75^{\circ} \mathrm{C}$ for $4.5 \mathrm{~h}$. After cooling at room temperature, the mixture was filtered and the solid washed with ethyl acetate. The filtrate was evaporated under reduced pressure, then purified by column chromatography using light petroleum ether/ethyl acetate $5 / 1$ as eluent to give compound 15. Yield $91 \%$. $[\alpha]_{\mathrm{D}}^{26}=-28.10(c$ $\left.0.33, \mathrm{CHCl}_{3}\right)$. Mp 67-68 ${ }^{\circ} \mathrm{C} .{ }^{1} \mathrm{H}$ NMR $(250 \mathrm{MHz}): \delta 3.81$ $(\mathrm{dd}, J=12.3$ and $6.6 \mathrm{~Hz}, 1 \mathrm{H}), 3.94(\mathrm{~d}, J=17.8 \mathrm{~Hz}, 1 \mathrm{H})$, $4.04(\mathrm{dd}, J=12.3$ and $7.4 \mathrm{~Hz}, 1 \mathrm{H}), 4.28(\mathrm{~d}, J=17.8 \mathrm{~Hz}$, $1 \mathrm{H}), 4.41(\mathrm{~d}, J=11.7 \mathrm{~Hz}, 1 \mathrm{H}), 4.70-4.81(\mathrm{~m}, 3 \mathrm{H}), 4.93$ $(\mathrm{d}, \quad J=5.6 \mathrm{~Hz}, 1 \mathrm{H}), 7.30-7.37(\mathrm{~m}, 5 \mathrm{H}) ;{ }^{13} \mathrm{C}$ NMR (62.5 MHz): $\delta$ 68.7, 72.3, 72.8, 82.5, 96.2, 127.9, 128.0, 128.5, 137.3, 208.4. IR (nujol) $1734, \mathrm{~cm}^{-1}$. Anal. Calcd for $\mathrm{C}_{12} \mathrm{H}_{14} \mathrm{O}_{4}$ : C, 64.85; H, 6.35. Found: $\mathrm{C}, 64.90 ; \mathrm{H}, 6.41$.

\section{Acknowledgements}

Financial support from the University of Palermo (funds for selected research topics) and Italian MIUR within the
National Research Project 'Non-aromatic heterocycles in stereocontrolled processes' is gratefully acknowledged.

\section{References}

1. Selected examples: (a) Rai, A. N.; Basu, A. Org. Lett. 2004, 6 , 2861-2863; (b) Roush, W. R.; Adam, M. A. J. Org. Chem. 1985, 50, 3752-3757; (c) Shibuya, H.; Kawashima, K.; Ikeda, M.; Kitagawa, I. Tetrahedron Lett. 1989, 30, 7205-7208; (d) Tuch, A.; Sanière, M.; Merrer, Y. L.; Depezay, J. C. Tetrahedron: Asymmetry 1996, 7, 897-906; (e) Metz, K.; Honda, M.; Komori, T. Liebigs Ann. Chem. 1993, 55-60; (f) Miyata, O.; Yamaguchi, S.; Ninomiya, I.; Naito, T.; Okamura, K. Chem. Pharm. Bull. 1996, 44, 636-638.

2. (a) Yoshino, T.; Nagata, Y.; Itoh, E.; Hashimoto, M.; Katoh, T.; Terashima, S. Tetrahedron 1997, 53, 10239-10252; (b) Rollins, S. B.; Williams, R. M. Tetrahedron Lett. 1997, 38, 4033-4036.

3. Takano, S.; Sugihara, T.; Satoh, S.; Ogasawara, K. J. Am. Chem. Soc. 1988, 110, 6467-6471.

4. (a) Spada, M. R.; Ubukata, M.; Isono, K. Heterocycles 1992, 34, 1147-1167; (b) Kim, K. S.; Cho, I. H.; Ahn, Y. H.; Park, J. I. J. Chem. Soc., Perkin Trans. 1 1995, 1783-1785; (c) Gravier-Pelletier, C.; Charvet, I.; Merrer, Y. L.; Depezay, J. C. J. Carbohydr. Chem. 1997, 16, 129-141.

5. (a) Chan, A. W. Y.; Ganem, B. Tetrahedron Lett. 1995, 36, 811-814; (b) Banwell, M.; De Savi, C.; Hockless, D.; Watson, K. Chem. Commun. 1998, 645-646.

6. (a) Inaba, T.; Birchler, A. G.; Yamada, Y.; Sagawa, S.; Yokota, K.; Ando, K.; Uchida, I. J. Org. Chem. 1998, 63, 7582-7583; (b) Inaba, T.; Yamada, Y.; Abe, H.; Sagawa, S.; Cho, H. J. Org. Chem. 2000, 65, 1623-1628.

7. (a) Cativela, C.; Diaz-de-Villegas, M. D.; Gálvez, J. A.; Garcia, J. I. Tetrahedron 1996, 52, 9563-9574; (b) Cativela, C.; Díaz-de-Villegas, M. D.; Galvez, J. A. Tetrahedron Lett. 1995, 36, 2859-2860; (c) Saito, S.; Takahashi, N.; Ishikawa, T.; Moriwake, T. Tetrahedron Lett. 1991, 32, 667-670; (d) Palomo, C.; Cabre, F.; Ontoria, J. M. Tetrahedron Lett. 1992, 33, 4819-4822.

8. Dequeker, E.; Compernolle, F.; Toppet, S.; Hoornaert, G. Tetrahedron 1995, 51, 5877-5890.

9. Fuji, K.; Kawabata, T.; kiryu, Y.; Sugiura, Y. Heterocycles 1996, 42, 701-722.

10. Sagawa, S.; Abe, H.; Hase, Y.; Inaba, T. J. Org. Chem. 1999, 64, 4962-4965.

11. Holt, K. E.; Leeper, F. J.; Handa, S. J. Chem. Soc., Perkin Trans. 1 1994, 231-234.

12. Han, H.; Cho, C. W.; Janda, K. D. Chem. Eur. J. 1999, 5, $1565-1569$.

13. Hobley, G.; Stuttle, K.; Wills, M. Tetrahedron 2003, 59, 4739-4748.

14. Lu, X.; Byun, H.-S.; Bittman, R. J. Org. Chem. 2004, 69 , 5433-5438, and references cited therein.

15. Flasche, M.; Scharf, H.-D. Tetrahedron: Asymmetry 1995, 6, 1543-1546.

16. (a) Gruttadauria, M.; Lo Meo, P.; Noto, R. Tetrahedron Lett. 2004, 45, 83-85; (b) Gruttadauria, M.; Lo Meo, P.; Riela, S.; D'Anna, F.; Noto, R. Tetrahedron: Asymmetry 2006, 17, 2713-2721; (c) Gruttadauria, M.; Riela, S.; Aprile, C.; Lo Meo, P.; D’Anna, F.; Noto, R. Adv. Synth. Catal. 2006, 348 , 82-92; (d) Riela, S.; Aprile, C.; Gruttadauria, M.; Lo Meo, P.; Noto, R. Molecules 2005, 10, 383-393; (e) Gruttadauria, M.; Aprile, C.; Lo Meo, P.; Riela, S.; Noto, R. Heterocycles 2004, 63, 681-690; (f) Aprile, C.; Gruttadauria, M.; Amato, M. E.; D’Anna, F.; Lo Meo, P.; Riela, S.; Noto, R. 
Tetrahedron 2003, 59, 2241-2251; (g) Gruttadauria, M.; Aprile, C.; Noto, R. Tetrahedron Lett. 2002, 43, 1669-1672; (h) Gruttadauria, M.; Aprile, C.; D'Anna, F.; Lo Meo, P.; Riela, S.; Noto, R. Tetrahedron 2001, 57, 6815-6822; (i) Gruttadauria, M.; Lo Meo, P.; Noto, R. Tetrahedron 2001, 57, 1819-1826; (j) Gruttadauria, M.; Aprile, C.; Riela, S.; Noto, R. Tetrahedron Lett. 2001, 42, 2213-2215.

17. Iacazio, G.; Martini, D.; Sanchez, S.; Faure, B. Tetrahedron: Asymmetry 2000, 11, 1313-1321.

18. Fadnavis, N. W.; Sharfuddin, M.; Kumara Vadivel, S. Tetrahedron: Asymmetry 2001, 12, 691-693.

19. Anilkumar, A. T.; Goto, K.; Takahashi, T.; Ishizaki, K.; Kaga, H. Tetrahedron: Asymmetry 1999, 10, 2501-2503.

20. Tiecco, M.; Testaferri, L.; Temperini, A.; Bagnoli, L.; Marini, F.; Santi, C. Chem. Eur. J. 2004, 10, 1752-1764.
21. Yamada, O.; Ogasawara, K. Synthesis 1995, 1291-1294.

22. Ema, T.; Yamaguchi, K.; Wakasa, Y.; Yabe, A.; Okada, R.; Fukumoto, M.; Yano, F.; Korenaga, T.; Utaka, M.; Sakai, T. J. Mol. Cat. B: Enzym. 2003, 22, 181-192.

23. For PCL (Pseudomonas Cepacea Lipase) structure see: (a) Kim, K. K.; Song, H. K.; Shin, D. H.; Hwang, K. Y.; Suh, S. W. Structure 1997, 5, 173-185; (b) Schrag, J. D.; Li, Y.; Cygler, M.; Lang, D.; Burgdorf, T.; Hecht, H.-J.; Schmid, R.; Schomburg, D.; Rydel, T. J.; Oliver, J. D.; Strickland, L. C.; Dunaway, C. M.; Larson, S. B.; Day, J.; McPherson, A. Structure 1997, 5, 187-202.

24. Ozanne, A.; Pouységu, L.; Depernet, D.; François, B.; Quideau, S. Org. Lett. 2003, 5, 2903-2906.

25. Barluenga, J.; Vázquez-Villa, H.; Ballesteros, A.; González, J. M. Org. Lett. 2002, 4, 2817-2819. 